symphysis after delivery was a little more mobile than formerly, but it did not dilate perceptibly during labour. In both these cases there appears to have been some permanent enlargement of the pelvis as a result of the previous symphysiotomy.

Tumours Complicating Pragnancy. $-\mathrm{Coe}^{9}$ reports several interesting cases of pregnancy complicated by uterine fibroids, and he points out that the rapidity of growth of a fibroid under the influence of pregnancy is in direct proportion to its more or less intimate relation to the uterus. Thus a pedunculated subperitoneal growth may remain unchanged, while an interstitial tumour undergoes marked enlargement. On the other hand, a subserous fibro-myoma situated in the lower uterine segment, or displaced and impacted in the pouch of Douglas may increase in size simply from obstruction to its circulation. Porter also lays stress on the position of the tumour, a growth in the lower segment of the uterus usually proving very serious. Gemmel $^{10}$ reports a case of hydatic cyst in the omentum which obstructed labour, and was subsequently removed by abdominal section. The patient was first seen after being in labour for 24 hours, and on examination a cystic tumour was felt low down in the pelvis; the first stage of labour was completed, and the head unable to descend into the pelvis. The tumour was the size of a cocoanut, and was, between the pains, felt to be movable; under an anæsthetic it was pushed out of the pelvis into the abdomen; after this forceps were applied to the head and the child easily delivered. The puerperium was normal. Six weeks later the abdomen was opened; both ovaries and Fallopiun tubes were found to be healthy, and the cyst, which contained jelly-like material and daughter cysts, was intimately connected with the omentum. W. S. Stone ${ }^{11}$ dezcribes a case of ovarian cystoma complicating preg- nancy which almost completely filled the pelvis, and in which he made a posterior vaginal incision and drained the cyst. It was unilocular, but could not be completely removed on account of the pregnancy. The patient made a good recovery.

Puerperal Insanity.-William Hirsch, ${ }^{12}$ writing on this subject, summarises the important points of the relation of child-bearing to mental diseases as follows: (1) A specific form of mental disease, which might be called puerperal insanity, does not exist. The different psychoses which are observed during any of the stages of gestation are the same as those we see in other patients; (2) pregnancy may, under certain circumstances, be one of the etiological factors of insanity. Its etiological importance, however, is proved by neither statistics nor clinical observation. It is, therefore, not permissible to terminate pregnancy on account of a psychosis, unless there are special indications for such intervention. (3) During parturition we sometimes meet with a passing disturbance of consciousness, the clinical features of which resemble psychical epilepsy. (4) Pyschoses which occur in connection with the act of parturition are produced $(a)$ by trauma in cases of difficult labour; (b) by anæmia and exhaustion, after severe hæmorrhage; and (c) by intoxication in septic cases, or cases with local inflammation, or uræmia. (5) Lactation, as such, plays no rôle at all in the production of insanity. It is due to other circumstances; that, during the first few months after delivery, women on the average are more predisposed to nervous and mental diseases than under ordinary conditions.

' Practitioner, Dec., 1899, Dr. Davis, 2 Brit. Med. Jour., Nov. 18, 1899. Abuladse. ${ }^{3}$ Brit. Med. Jour., Dec. 9, 1899 , Levniovitsch. 4 Lancet, Dec. 23, 1899, Dr. Everke. 5 Brit. Med. Jour., Dec. 30, 1899, Vorhees. 6 Brit. Med. Jour., Dec. 9, 1899, Hillmann. 7 Brit. Med. Jour., Oct. 14, 1899, Whitridge Wilkiams. 8 Scottish Med. and Surg. Jour., Jan., 1900, R. O. Buist. 9 New York Med. Jonr., Nov. 25, 1899, Ooe. io Brit. Med. Jour., Dec. 9, 1899, Gemmel. "11 Med. Rec., Nov. 11, 18.9, W. S. Stone, 12 Med. Rec., Jan. 6, 1900, William Hirsch.

\title{
PROGRESS IN OTOLOGY.
}

Otitis Media.-Marcel Lermoyez, ${ }^{1}$ as the result of a number of observations, is firmly convinced that otitis media is often the result of contagion, not of an obvious contagion, but of one which is insidious and easily escapes observation. He briefly describes seven, out of a total number of 20 cases of acute otitis media treated by him in two years, in which inflammation of the same type was apparently developed as a result of contagion. The patients infected one another by the clasical naso-tubal route; they commenced necessarily by having a coryza, but this was so attenuated as to pass unperceived. The cases were of secondary otitis. The observations tended to prove that "given the first patient affected with influenza complicated with otitis, any other influenzal patient put in contact with him will run a great risk of acquiring this otitic complication." Statistics are given showing the frequency of secondary otitis in hospital, its rarity in private practice. Isolation of patients, especially children, with acute otitis is therefore strongly recommended.

Discussing the therapy of otitis media, Goldstein ${ }^{1}$ divided the subject into two sections: (1) Where the membrana tympani was intact; and (2) where perforations of the tympanic membrane existed. The catarrhal and non-suppurative forms came in the first group, the suppurative form in the latter. The therapy of the catarrhal form had varied much in recent years. It included such procedures as inflation of the tympanum with the air-bag of Politzer, or with the Eustachian catheter ; injections of fluid medicaments in an aqueous or an oleaginous menstruum; injection of pepsin through the drum membrane with a hypodermic syringe; the introduction of vapours of ammonium chloride, chloroform, \&c.; the use of the pressure probe of Lucae, or of the Eustachian bougie ; and massage of the drum membrane by the hand-masseur or the electric masseur engine. The results were only moderately good. As atrophic rhinitis might be considered the sequel of the hypertrophic form, so sclerotic or adhesive otitis might be regarded as the sequel of hypertrophic otitis. Goldstein had used purified petroleum as a base for his medications, and had found iodine, menthol, carbolic acid, eucalyptus, \&c., the most useful agents. If the Eustachian tube was free, as shown by the auscultation tube, the fluid was injected through the Eustachian tube. Where that offered obstruction, he had used continued vapour inflations with good results. For this purpose he employed a handnebuliser with a glass nasal tip. Following the vaporisation or injection, he used the aural masseur. He believed that the treatment of chronic purulent otitis by irrigation through the meatus was 
contra-indicated where there was a large perforation, and ready entrance of the fluid into the tympanum was provided. Infection of the attic or antrum might be caused by driving infectious material into them with the syringe. Again, it was irritation of the sodden mucous membrane by fluids which led to the growth of polypi which the addition of aqueous fluids aggravated. The indiscriminating use of the nasal douche frequently led to infection of the tympanum. H. A. Alderton ${ }^{2}$ advocates strongly the use of syringing in these cases, at least a pint of liquid every two hours being recommended. An antiseptic is not necessary if the canal is thoroughly dried, as the solution never reaches the middle ear. He regards the chances of forcing infected material into the mastoid cells as not worthy of consideration in contrast with the benefits derived. In all cases of otitis media in which the mastoid did not show any traces of inflammation during life pus was found post-mortem in the mastoid cells. Powders were unsuitable in acute suppuration; they tended to pack in front of the drum and impeded drainage, and did not reach the inflamed surface. Stetter ${ }^{3}$ supports Lucae in the latter's statement that the general symptoms calling for the radical operation for otitis are marked dizziness with disturbances in gait, nausea, and romiting; while the local symptoms pointing that way have to do with the duration of the disease, the condition of the post-aural region, the pain, the facial paralysis, and the nature and quality of the discharge. The value of iodide of potash in rendering the thick tenacious pus more fluid so that the ear can be properly cleansed is pointed out. Also Hang's chinolin.naphthol gauze as a packing is recommended. It is very soft and of open mesh, and can be packed even into the middle ear itself. Pure trifor dis overcoming the fœetid and stinking condition of the discharge menthoxol with an equal part of water is praised. In contact with pus it gives off oxygen, and leaves behind menthol dissolved in alcohol. It has been very successful. Urban Pritchard ${ }^{4}$ has contributed a paper on the antiseptic purification of the meatus for operations, and as a treatment in middle ear suppura. tions. The treatment is specially adapted to cases such as the following: (1) When there is no suppuration and tions meman tympani is intact, for it allows of operathere without infection of deeper parts. (2) When It is not perforation and suppuration already present. very septic cases. The mode of procedure in the first
. The class-e.g., in preparing for removal of an exostosis, or for any operation, is to syringe well with 1 in 40 carbolic, and then mop out with 1 in 20 until the parts are quite clean, so far as the eye can judge. This is done an hour before operation. The whole auricle is then well serubbed with 1 in 20 , and then the meatus lightly packed with a strip of double cyanide gauze, wrung out of a 1 in 20 or 40 carbolic to get rid of the irritating soluble cyanides, and a pad of similar gauze is fixed over the auricle. The operation is then conducted with all the antiseptic precautions used in ordinary surgery, and if anything needs removing it is done by syringing with 1 in 40 carbolic, the syringe being perfectly purified first. Ordinary antiseptic dressings are used after the operation. In paracentesis in acute otitis suppuration may often be altogether prevented if it has not already occurred, which is frequently the case. In cases where suppuration already exists, e.g., where polypi have to be removed, or carious spots curetted, the ear should be syringed out with 1 in 40 carbolic once or twice a day for a week before the operation. The syringing must be repeated after the operation, when frequently epidermic caseous masses will be dislodged. If the case is very septic the strip of gauze should be tipped with iodoform powder. Granulations and polypi treated in this way yield often excellent results. 'The gauze packings have to be repeated once a day to once in two, three, or more days according to the conditions present. In chronic otorrhoa, even after the discharge has ceased, it is well to continue the dressing of gauze, using a superficial and a deep piece, without a bandage, so that if the patient works out the superficial piece the other still remains undisturbed. Lysol or perchloride of mercury might be substituted for carbolic in some cases. E. B. Gleason ${ }^{5}$ contributes a paper on the silver salts in the treatment of chronic suppuration of the middle ear. The use of nitrate of silver in these cases had not been remarkably successful, as the irritating effects had overshadowed the antiseptic and astringent properties. Certain organic salts of silver have been stated to act as astringents for mucous membranes without being in the least irritating. This led Gleason to think they might prove very useful in treating prolonged otorrhœas in which the attic and probably the mastoid autrum were involved. Protargol was therefore tried in a 5 per cent. solution injected as high up into the attic as possible by means of a Blake's cannula. Then the parts were massaged with Siegle's pneumatic speculum in order to force the solution into more distant parts than could be reached with the syringe. Before this the middle ear had been cleansed and dried. Four attic cases are detailed, in three of which the treatment was highly successful. The solution is quite unirritating to the middle-ear mucous membrane.

1 Vide Jour. Laryng., Rhinol, and Otol., Dec., 1899. 2 Laryngoscope, Jan., 1900. ' and Otol., Mar., 1900, 5 Laryngoscope, Mar., 1900.

\section{NEW APPLIANCES AND THINGS MEDICAL. \\ [Wo shall be glad to reW APPLIANCES AND THINGS MEDICAL \\ preparations and appliances which may be brought out from time to time.]}

SOAPS AND TOILET PREPARATIONS.

WE the above firm samples of several different soaps from perties will be. Those with antisepti; and medicinal proCook's will be of most interest to readers of THE Hospital. germicidald medal antiseptic soap possesses remarkable from a properties, which is naturally to be expected potassium which contains biniodide of mercury and soap basis. The biniodide is skilfully combined with the skin diseas in such a way as to be chemically available. For very useful, which are clearly of microbic origin this soap is purposes. surgeses. As an antiseptic soap for cleaning the hands of feons or the skin of patients before operation it is to be highly recommended. Cook's sulphur and glycerine soap is a soothing variety for delicate skins, and in certain forms of eczema and mild psoriasis it can be applied with advantage. The toilet carbolic soap and the compressed coal tar are both preparations which are to be commended where antiseptic properties are sought for in a mild though effective degree. As a really luxurious toilet soap of most delicious perfume there is nothing to beat the Russian Violet, but, though luxurious to a degree, it is perfectly harmless to the most delicate skin, with fine emollient properties. Cook's Riviera soap is a delightfully-scented soap for ordinary toilet purposes ; it is particularly pure and economical in use. Their cold cream and oatmeal soap should be used when the water is hard, and is a very successful kind for nursery use. 